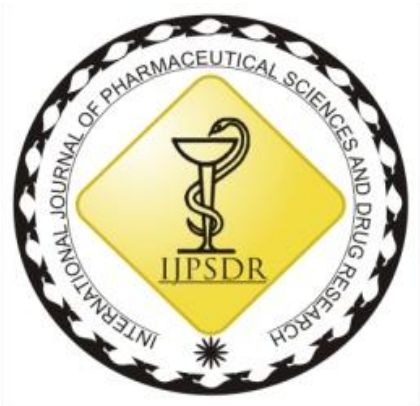

ISSN: 0975-248X

RESEARCH ARTICLE CODEN (USA): IJPSPP

$($ (c) $)$ EY-NC-SA

\title{
Anti-diabetic Activity of Few India Indigenous Medicinal Plants
}

\author{
Rajesh Nagar*, Rakesh Patel \\ Faculty of Pharmacy, Dr. A. P. J. Abdul Kalam University, Indore, Madhya Pradesh, India
}

Copyright (C) 2019 Rajesh Nagar et al. This is an open access article distributed under the terms of the Creative Commons AttributionNonCommercial-ShareAlike 4.0 International License which allows others to remix, tweak, and build upon the work non-commercially, as long as the author is credited and the new creations are licensed under the identical terms.

\begin{abstract}
The traditional system of medicine is so ingrained in our culture that, about $75 \%$ of the Indian population depends on this indigenous system for relief. With such a huge section of an ever increasing population relying on herbal remedies, it is imperative that the plant products which have been in use for such a long time be scientifically supported for their efficacy. Diabetes a metabolic disorders is most frequent occurring disease in India. The present paper reports with the investigation of antidiabetic potential of Eugenia jambolana (Bark), Phyllanthus niruri (Whole plant), Momordica charantia Linn. (Seeds) and Nyctanthes arbor-tristis (Leaves).
\end{abstract}

Keywords: Diabetes, Antidiabetic, Indigenous, herbal remedies.

DOI: 10.25004/IJPSDR.2019.110601

Int. J. Pharm. Sci. Drug Res. 2019; 11(6): 278-283

*Corresponding author: Mr. Rajesh Nagar

Address: Faculty of Pharmacy, Dr. A. P. J. Abdul Kalam University, Indore, Madhya Pradesh, India

E-mail $\bowtie$ : rnagar@gmail.com

Relevant conflicts of interest/financial disclosures: The authors declare that the research was conducted in the absence of any commercial or financial relationships that could be construed as a potential conflict of interest.

Received: 05 July, 2019; Revised: 19 October, 2019; Accepted: 27 October, 2019; Published: 28 November, 2019

\section{INTRODUCTION}

Diabetes is a chronic disorder of carbohydrate, fat and protein metabolism characterized by increased fasting and post prandial blood sugar levels. The global prevalence of diabetes is estimated to increase, from $4 \%$ in 1995 to $5.4 \%$ by the year 2025 . WHO has predicted that the major burden will occur in developing countries. Studies conducted in India in the last decade have highlighted that not only is the prevalence of diabetes high but also that it is increasing rapidly in the urban population. It is estimated that there are approximately 33 million adults with diabetes in India. This number is likely to increase to 57.2 million by the year 2025. [1-3]

In the last few years there has been an exponential growth in the field of herbal medicine and these drugs are gaining popularity both in developing and developed countries because of their natural origin and less side effects. Many traditional medicines in use are derived from medicinal plants, minerals and organic matter. A number of medicinal plants, traditionally used for over 1000 years named rasayana are present in herbal preparations of Indian traditional health care systems. In Indian systems of medicine most practitioners formulate and dispense their own recipes. The World Health Organization (WHO) has listed 21,000 plants, which are used for medicinal purposes around the world. Among these 2500 species are in India, out of which 150 species are used commercially on a fairly large scale. India is the largest producer of medicinal herbs and is called as botanical garden of the world. [4-6] The present work was aimed on development of standardization parameters of selected herbs used in the treatment of diabetes mellitus, a 
major crippling disease in the world leading to huge economic losses.

\section{MATERIALS AND METHODS}

Collection of herbs and their authentication

The plant parts viz., EJB: Eugenia jambolana (Bark), PNWP: Phyllanthus niruri (Whole plant), MCS: Momordica charantia Linn. (Seeds) and NATL: Nyctanthes arbor-tristis (Leaves) were collected in the months of July-September 2016 from the various local sites of Malwa region of Madhya Pradesh and identified \& authenticated by Dr. S. N. Dwivedi, Prof. and Head, Department of Botany, Janata PG College, A. P. S. University, Rewa, (M.P.) and was deposited in our Laboratory.

Pharmacological screening [7-10]

Procurement of experimental animals

The mice were used for acute toxicity study as per OECD guidelines 423. The animals were fed with standard pellet diet (Hindustan lever Ltd. Bangalore) and water ad libitum. All the animals were housed in polypropylene cages. The animals were kept under alternate cycle of 12 hours of darkness and light. The animals were acclimatized to the laboratory condition for 1 week before starting the experiment. The experimental protocols were approved by Institutional Animal Ethics Committee after scrutinization.

\section{Experimental animals}

The Wister strains of male albino rats weighing between 100 and $150 \mathrm{~g}$ were obtained for the present study, from Govt. Veterinary Hospital, Mhow, Madhya Pradesh. The animals were housed in larger spacious cages and they were fed with commercial pelleted rat chow marketed by Hindustan Lever Ltd., Bangalore, India, under the trade name Gold Mohur Rat Feed and had free access to water ad labium. The animals were well acclimatized to standard environmental conditions of temperature and $12 \mathrm{~h}$ light dark cycles throughout the experimental period. The animals used in the present study were approved by the Institutional Animal Ethical Committee.

\section{Primary Screening for Diabetes}

Oral glucose tolerance test (OGTT)

Oral glucose tolerance tests (OGTT) were carried out as per the procedure previously described by Joy and Kuttan with minor modifications. Briefly, fasted mice were grouped into five groups of six mice each. The various groups received different treatments like Group 1 received vehicle (1\% Tween 20 in water, 10 $\mathrm{ml} / \mathrm{kg}$ body weight) and served as control, Group 2 received standard drug (glibenclamide, $10 \mathrm{mg} / \mathrm{kg}$ body weight). Groups 3-18 received, respectively, extract at doses of $200 \mathrm{mg}$ per $\mathrm{kg}$ body weight. All substances were orally administered by gavaging. The amount of Tween 20 administered was same in both control and experimental mice. Following a period of one hour as described earlier, all mice were orally administered $2 \mathrm{~g}$ glucose per $\mathrm{kg}$ of body weight. Blood samples were collected 120 minutes after the glucose administration through puncturing heart following previously published procedures. Blood glucose levels were measured with a glucometer. The percent lowering of blood glucose levels were calculated according to the formula described below. Percent lowering of blood glucose level $=(1-\mathrm{We} / \mathrm{Wc}) \times 100$, where We and Wc represents the blood glucose concentration in glibenclamide or extract administered mice (Groups 2, 3-18), and control mice (Group 1), respectively.

\section{Statistical analysis}

Experimental values are expressed as mean \pm SEM. Independent Sample t-test was carried out for statistical comparison. Statistical significance was considered to be indicated by a $p$ value $<0.05$ in all cases.

Secondary Screening for anti-diabetic activity

Preparation of alloxan monohydrates

Alloxan was prepared by weighing $1 \mathrm{~g}$ of alloxan and dissolving in $20 \mathrm{ml}$ of water for injection. Alloxan at this calculated dose is said to have a concentration of 50 $\mathrm{mg} / \mathrm{ml}$.

\section{Hypoglycemic Activity}

Different groups of each six rats were used in the present investigation. The basal concentration of blood glucose level of all the animals was recorded and 6 animals were separated to serve as normal control. The remaining animals received a single injection of Alloxan monohydrate in water for injection at a dose of $150 \mathrm{mg} / \mathrm{kg}$ bodyweight given by intra-peritoneal route. After 4 days of Alloxan administration, the blood glucose was estimated and animals with blood glucose levels in the range $280 \mathrm{mg} / \mathrm{dl}$ and $380 \mathrm{mg} / \mathrm{dl}$ were selected and divided into groups.

Group 1:- Untreated control (Normal saline water)

Group 2:- Diabetic control (Alloxan 150 mg/kg)

Group 3:- Diabetic+ Glibenclamide $(10 \mathrm{mg} / \mathrm{kg})$

Group 4:- Diabetic + EEEJB (500 mg)

Group 5:- Diabetic + AEEJB (500 mg

Group 6:- Diabetic + EEPNWP (500 mg)

Group 7:- Diabetic + AEPNWP (500 mg)

Group 8:- Diabetic + EEMCS (500 mg)

Group 9:- Diabetic + AEMCS (500 mg)

Group 10:- Diabetic + EENATL (500 mg)

Group 11:- Diabetic + AENATL (500 mg)

Statistical analysis

Data were analyzed by comparing values for different treatment groups with the values for individual controls. The significant differences among values were analyzed using analysis of variance (one-way ANOVA) in latest computer software programme. All the obtained results are expressed as $X$ (Mean) \pm SEM, $n=6$. (One way ANOVA followed by Bonferroni multiple comparison test).

\section{RESULTS AND DISCUSSION}

The PEE, CE, EE and AE of EJB: Eugenia jambolana (Bark), PNWP: Phyllanthus niruri (Whole plant), MCS: Momordica charantia (Seeds) and NATL: Nyctanthes arbor-tristis (Leaves) were screened for acute toxicity study by OECD guideline no. 423 for determination of 
LD50. The results showed that the PEE, CE, EE and AE of EJB: Eugenia jambolana (Bark), PNWP: Phyllanthus niruri (Whole plant), MCS: Momordica charantia (Seeds) and NATL: Nyctanthes arbor-tristis (Leaves) were belonging to category-5 (unclassified). Hence, $\mathrm{LD}_{50}$ was $5000 \mathrm{mg} / \mathrm{kg}$, therefore, ED 50 was $500 \mathrm{mg} / \mathrm{kg}$. Therefore doses of $500 \mathrm{mg}$ were selected for present investigation. In oral glucose tolerance tests, ethanolic and aqueous extract of EJB: Eugenia jambolana (Bark), PNWP: Phyllanthus niruri (Whole plant), MCS: Momordica charantia (Seeds) and NATL: Nyctanthes arbor-tristis (Leaves) was found to be significant and dosedependently reduced blood glucose levels in glucoseloaded mice by more than $30 \%$ at the dose of $500 \mathrm{mg}$ per $\mathrm{kg}$ body weight in mice. When the comparison was made with standard antihyperglycemic drug (glibenclamide) reduced blood glucose levels by $41.31 \%$ at a dose of $10 \mathrm{mg}$ per $\mathrm{kg}$ bw it was found that PEE and CE of EJB: Eugenia jambolana (Bark), PNWP: Phyllanthus niruri (Whole plant), MCS: Momordica charantia (Seeds) and NATL: Nyctanthes arbor-tristis (Leaves) was not effective as glibenclamide in reducing blood glucose.

Another important aspect of this study is that to our knowledge this is the first description of the ability of EJB: Eugenia jambolana (Bark), PNWP: Phyllanthus niruri (Whole plant), MCS: Momordica charantia (Seeds) and NATL: Nyctanthes arbor-tristis (Leaves) to reduce blood glucose. If this holds up for the active component(s) along with their mechanism of action is identified and elucidated, this can be a highly promising means towards alleviation of high blood glucose levels in diabetic patients or people with impaired glucose metabolism.

Table 1: Effect of extracts on blood glucose level in hyperglycemic mice following 120 minutes of glucose loading

\begin{tabular}{cccc}
\hline Treatment & $\begin{array}{c}\text { Dose } \\
(\mathbf{m g} / \mathbf{k g} \text { bw) }\end{array}$ & $\begin{array}{c}\text { Blood glucose } \\
\text { level }(\mathbf{m m o l} / \mathbf{l})\end{array}$ & $\begin{array}{c}\text { \% lowering of } \\
\text { blood glucose level }\end{array}$ \\
\hline Control & $10 \mathrm{ml}$ & $5.93 \pm 0.18$ & - \\
Glibenclamide & $10 \mathrm{mg}$ & $3.48 \pm 0.08$ & 41.31 \\
PEEEJB & $500 \mathrm{mg}$ & 5.67 & 4.38 \\
CEEJB & $500 \mathrm{mg}$ & 4.88 & 17.70 \\
EEEJB & $500 \mathrm{mg}$ & 4.14 & 30.18 \\
AEEJB & $500 \mathrm{mg}$ & 3.93 & 33.72 \\
PEEPNWP & $500 \mathrm{mg}$ & 5.51 & 7.08 \\
CEPNWP & $500 \mathrm{mg}$ & 5.10 & 13.99 \\
EEPNWP & $500 \mathrm{mg}$ & 4.13 & 30.35 \\
AEPNWP & $500 \mathrm{mg}$ & 4.02 & 32.20 \\
PEEMCS & $500 \mathrm{mg}$ & 4.41 & 25.63 \\
CEMCS & $500 \mathrm{mg}$ & 4.43 & 25.29 \\
EEMCS & $500 \mathrm{mg}$ & 4.10 & 30.86 \\
AEMCS & $500 \mathrm{mg}$ & 3.94 & 33.55 \\
PEENATL & $500 \mathrm{mg}$ & 5.32 & 10.28 \\
CENATL & $500 \mathrm{mg}$ & 5.34 & 9.94 \\
EENATL & $500 \mathrm{mg}$ & 4.35 & 26.64 \\
AENATL & $500 \mathrm{mg}$ & 4.12 & 30.52
\end{tabular}

All administrations were made orally only. Values are expressed as Mean \pm SEM, $(\mathrm{n}=6) ; p<0.05$

From the results obtained of OGTT for next step i.e., Secondary Screening for anti-diabetic activity only ethanolic and aqueous extract of EJB: Eugenia jambolana (Bark), PNWP: Phyllanthus niruri (Whole plant), MCS:
Momordica charantia (Seeds) and NATL: Nyctanthes arbor-tristis (Leaves) were chosen.

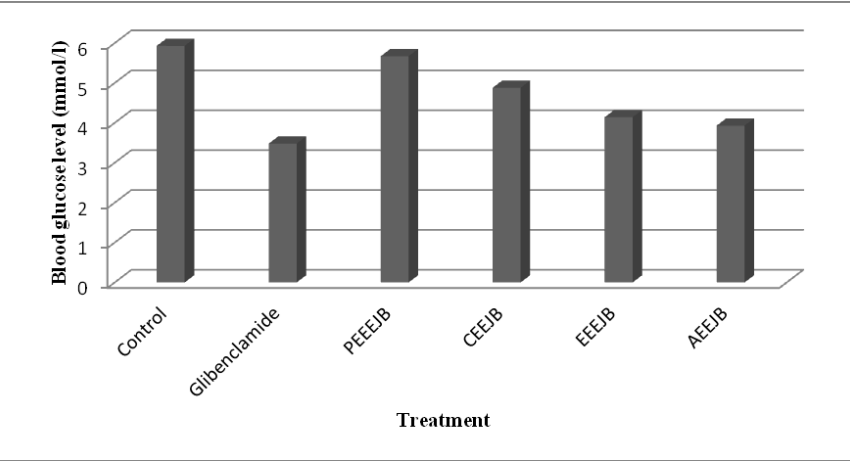

Graph 1: OGTT for various extract of EJB: Eugenia jambolana (Bark)

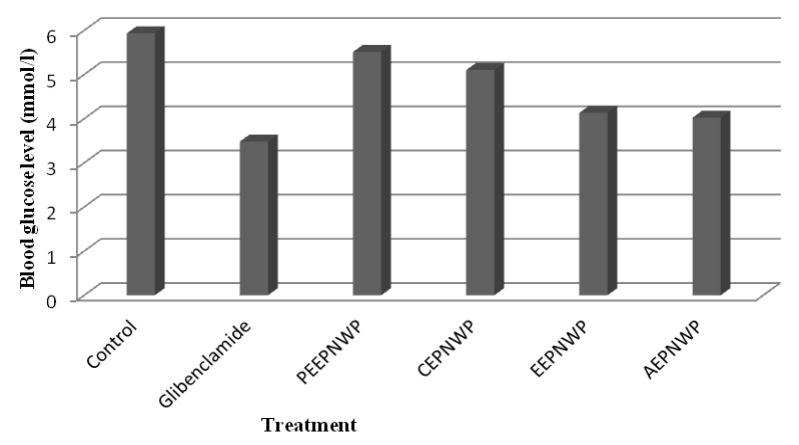

Graph 2: OGTT for various extract of PNWP: Phyllanthus niruri (Whole plant)

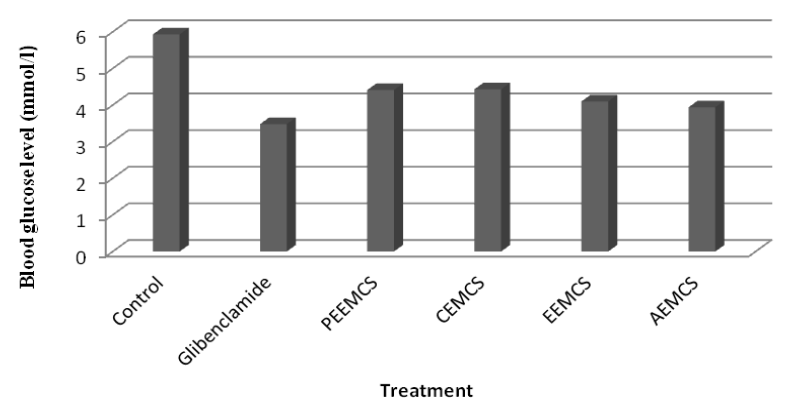

Graph 3: OGTT for various extract of MCS: Momordica charantia (Seeds)

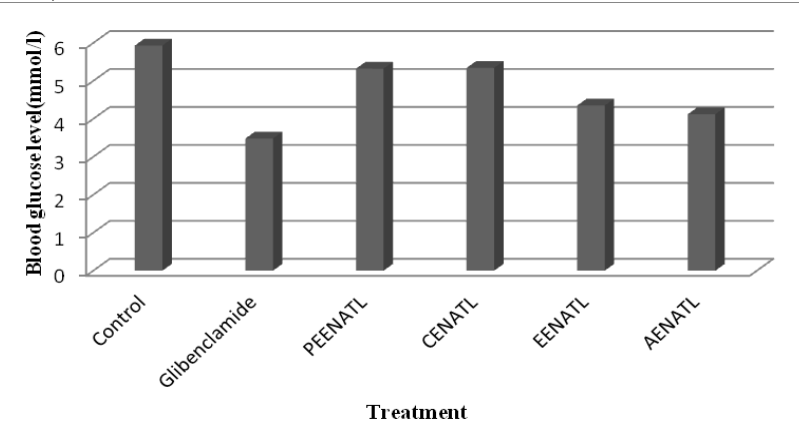

Graph 4: OGTT for various extract of NATL: Nyctanthes arbortristis (Leaves)

From the results obtained of OGTT for next step i.e., Secondary Screening for anti-diabetic activity only ethanolic and aqueous extract of EJB: Eugenia jambolana (Bark), PNWP: Phyllanthus niruri (Whole plant), MCS: Momordica charantia (Seeds) and NATL: Nyctanthes arbor-tristis (Leaves) were chosen. In the present 
investigation the data of the blood glucose level of rats treated with Alloxan (150 mg/kg body weight) produced diabetes within 72 hours. After 72 hours of Alloxan administered the blood glucose levels of rats were observed.

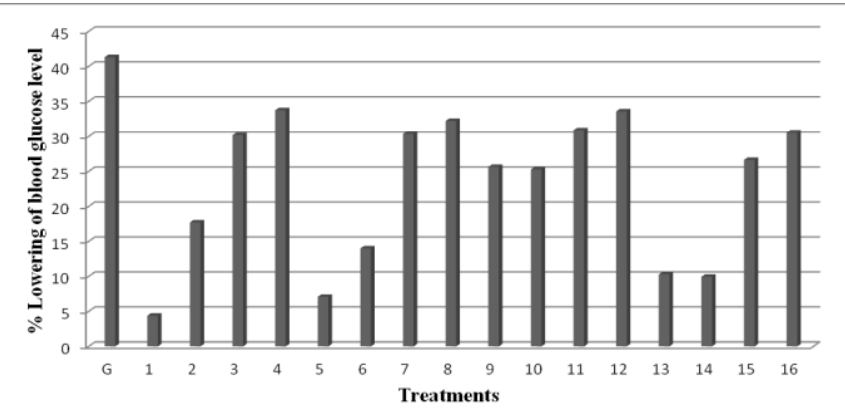

Graph 5: Percentage lowering of blood glucose level in various extracts

Abbr.: G=Glibenclamide; 1- PEEEJB, 2= CEEJB; 3= EEEJB, 4= AEEJB; $5=$ PEEPNWP; $6=$ CEPNWP, $7=$ EEPNWP; $8=$ AEPNWP; $9=$ PEEMCS; $10=$ CEMCS; 11= EEMCS; $12=$ AEMCS; $13=$ PEENATL; $14=$ CENATL $; 15=$ EENATL; $16=$ AENATL

Table 2 indicates effect of administration of feeding the ethanolic and aqueous extract of EJB: Eugenia jambolana (Bark), PNWP: Phyllanthus niruri (Whole plant), MCS: Momordica charantia (Seeds) and NATL: Nyctanthes arbor-tristis (Leaves) on body weight and fluid intake in normal and diabetic rats. Table 3 showed effect of administration of feeding the ethanolic and aqueous extract of EJB: Eugenia jambolana (Bark), PNWP: Phyllanthus niruri (Whole plant), MCS: Momordica charantia (Seeds) and NATL: Nyctanthes arbor-tristis (Leaves) on total hemoglobin and urine sugar in normal and diabetic rats.

It was observed in that significant lowering of sugar in aqueous extract of all the extracts i.e., EJB: Eugenia jambolana (Bark), PNWP: Phyllanthus niruri (Whole plant), MCS: Momordica charantia (Seeds) and NATL: Nyctanthes arbor-tristis (Leaves). The administration of AEEJB \& AEMCS at a dose of $500 \mathrm{mg} / \mathrm{kg}$ body weight showed significant anti-hyperglycaemic effect (117.07 \pm $1.25 \& 119.71 \pm 1.12)$ at $21^{\text {st }}$ day which was evident from the $1^{\text {st }}$ day on wards as compared to standard (112.72 \pm 1.04). The aqueous extract showed better efficacy than the ethanolic extract in all the treated extract. The antihyperglycaemic effect of the extract on the fasting blood sugar levels on diabetic rats is shown in table 4 . The decreasing blood glucose levels are comparable with that of $10 \mathrm{mg} / \mathrm{kg}$ of Glibenclamide. The blood glucose levels of the anti-diabetic activity of alloxan induced diabetic rats were shown in table 3 . It represents that decrease in blood glucose levels. The Glibenclamide (10 mg/kg body weight) shows significant effect on compare to the initial and more significant effect on the $7^{\text {th }}$ Day compare to the initial.

Table 2: Effect of administration of feeding the ethanolic and aqueous extract of selected herbs on body weight and fluid intake in normal and diabetic rats

\begin{tabular}{cccc}
\hline \multirow{2}{*}{ Group } & \multicolumn{2}{c}{ Body Weight $(\mathbf{g})$} & \multirow{2}{*}{ Fluid intake g/animal/day } \\
\cline { 2 - 4 } Control & Before treatment & After treatment & $21.41 \pm 0.47$ \\
Diabetic control & $192 \pm 1.08$ & $221.5 \pm 1.39$ & $74.01 \pm 0.01^{\# \# \#}$ \\
Standard (10mg/ $\mathrm{kg})$ & $202.16 \pm 1.35$ & $168.5 \pm 2.513^{\# \# \#}$ & $54.02 \pm 0.15^{* * *}$ \\
EEEJB (500 mg) & $206.06 \pm 1.72$ & $221.72 \pm 1.16^{* * *}$ & $58.92 \pm 0.01^{* * *}$ \\
AEEJB (500 mg) & $208.13 \pm 1.03$ & $221.06 \pm 1.02^{* * *}$ & $57.12 \pm 0.10^{* * *}$ \\
EEPNWP (500 mg) & $196.39 \pm 2.02$ & $212.1 \pm 1.16^{* * *}$ & $56.17 \pm 0.39^{* * *}$ \\
AEPNWP (500 mg) & $204.41 \pm 2.01$ & $221.03 \pm 2.18^{* * *}$ & $56.13 \pm 0.15^{* * *}$ \\
EEMCS (500 mg) & $197.02 \pm 2.31$ & $216.06 \pm 2.12^{* * *}$ & $70.11 \pm 1.20$ \\
AEMCS (500 mg) & $211.29 \pm 2.10$ & $177.30 \pm 1.12$ & $68.45 \pm 1.01$ \\
EENATL (500 mg) & $191.02 \pm 1.01$ & $158.11 \pm 1.02$ & $57.82 \pm 0.17^{* *}$ \\
AENATL (500 mg) & $204.29 \pm 1.18$ & $187.12 \pm 1.11^{* *}$ & $60.19 \pm 0.33^{* *}$
\end{tabular}

All values are expressed as mean \pm S.E.M $(\mathrm{n}=6),{ }^{* * *} P<0.001$ as compared diabetic control (normal saline), ${ }^{* *} P<0.01$ as compared diabetic control (normal saline), $\# \# P<0.001$ as compared to Control. One-way ANOVA followed by Bonferroni multiple comparison test

The results of histopathology of pancreas (Fig. 1) of aqueous extract are as mentioned below:

Control: Normal architecture with acini of serous epithelial cells along with nest of endocrine cells separated by fibrocollagenous stroma into lobules. No fibrosis or any inflammation.

Diabetic control: Normal architecture with acini of serous epithelial cells along with nest of endocrine cells separated by fibrocollagenous stroma into lobules. Focal lymphocytic infiltrate present in stroma.

Standard: The normal architecture was restored to the same as that of the standard drug treated pancreas.

AEEJB: Normal architecture with acini of serous epithelial cells along with nest of endocrine cells separated by fibrocollagenous stroma into lobules. No fibrosis or any inflammation seen.

AEPNWP: Normal architecture with acini of serous epithelial cells along with nest of endocrine cells separated by fibrocollagenous stroma into lobules. No fibrosis or any inflammation seen.

AEMCS: Normal architecture with acini of sepithelial cells along with nest of endocrine cells separated by fibrocollagenous stroma into lobules. No fibrosis or but little inflammation seen.

AENATL: Normal architecture with acini of serous epithelial cells along without nest of endocrine cells separated by fibrocollagenous stroma into lobules. Less fibrosis or little inflammation seen. 

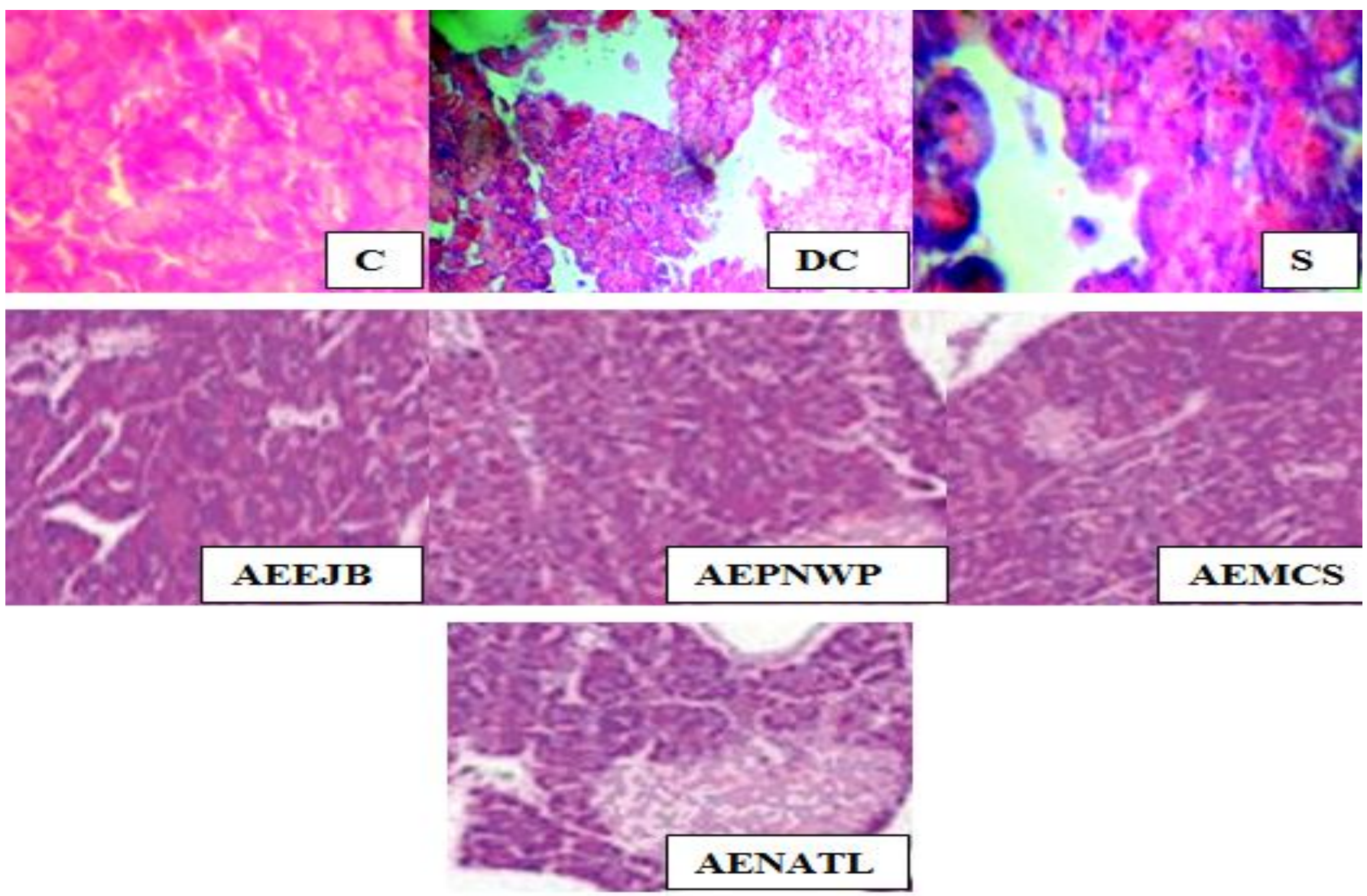

Fig. 1: Histopathology of pancreas of aqueous extract of selected herb

Table 3: Effect of administration of feeding the ethanolic and aqueous extract of selected herb on total haemoglobin and urine sugar in normal and diabetic rats

\begin{tabular}{ccccc}
\hline \multirow{2}{*}{ Group } & \multicolumn{2}{c}{ Total hemoglobin (\%) } & \multicolumn{2}{c}{ Urine sugar } \\
\cline { 2 - 5 } Control & Before treatment & After treatment & Before treatment & After treatment \\
\hline Diabetic control & $11.53 \pm 0.12$ & $11.7 \pm 0.481$ & Nil & +4 \\
Standard $(10 \mathrm{mg} / \mathrm{kg})$ & $12.82 \pm 0.11$ & $7.69 \pm 0.744^{\# \# \#}$ & +4 & +4 \\
EEEJB $(200 \mathrm{mg})$ & $13.13 \pm 0.67$ & $16.25 \pm 0.399^{* * *}$ & +4 & +2 \\
EEEJB $(500 \mathrm{mg})$ & $13.09 \pm 0.23$ & $15.97 \pm 0.154^{* * *}$ & +4 & +2 \\
AEEJB (500 mg) & $13.06 \pm 0.14$ & $16.23 \pm 0.216^{* * *}$ & +4 & +2 \\
EEPNWP (500 mg) & $14.06 \pm 0.19$ & $13.61 \pm 0.297^{* * *}$ & +4 & +2 \\
AEPNWP (500 mg) & $13.72 \pm 0.18$ & $16.5 \pm 0.196^{* * *}$ & +4 & +4 \\
EEMCS (500 mg) & $14.23 \pm 0.16$ & $9.098 \pm 0.256$ & +4 & +4 \\
AEMCS (500 mg) & $14.91 \pm 0.82$ & $8.987 \pm 0.142$ & +4 & +4 \\
EENATL (500 mg) & $12.19 \pm 0.21$ & $8.234 \pm 0.276$ & +4 & +4 \\
\hline
\end{tabular}

All values are expressed as mean \pm S.E.M $(n=6),{ }^{* * *} P<0.001$ as compared diabetic control (normal saline), ${ }^{* *} P<0.01$ as compared diabetic control (normal saline), ${ }^{\# \# P} P<0.001$ as compared to Control. One-way ANOVA followed by Bonferroni multiple comparison test

Table 4: Effect of administration of feeding the ethanolic and aqueous extract extract of selected herb on serum glucose estimation in normal and diabetic rats

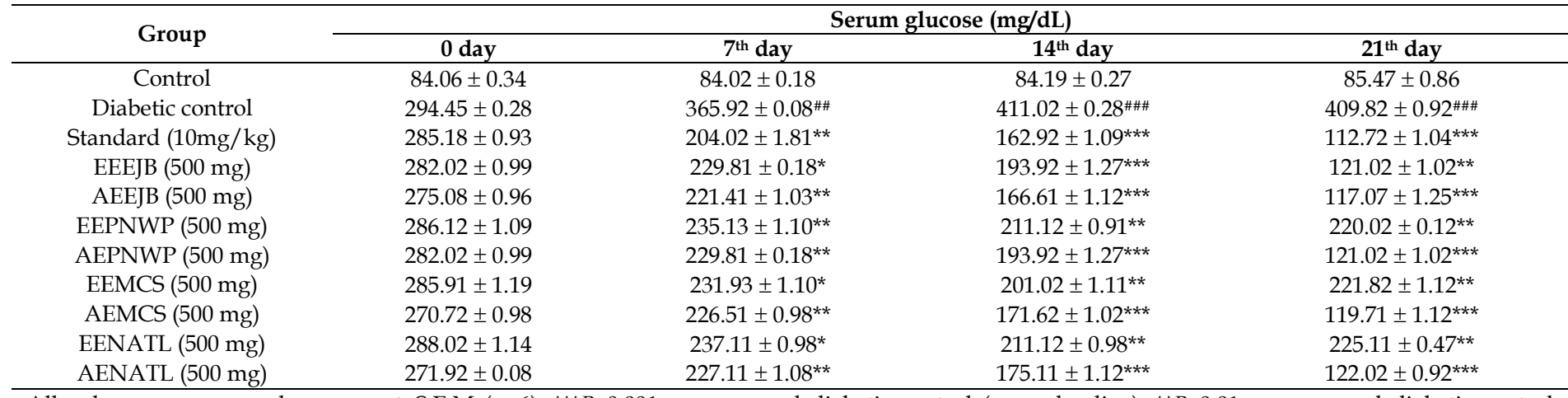

All values are expressed as mean \pm S.E.M $(n=6),{ }^{* * *} P<0.001$ as compared diabetic control (normal saline), ${ }^{* *} P<0.01$ as compared diabetic control (normal saline), $\# \# P<0.001$ as compared to Control. One-way ANOVA followed by Bonferroni multiple comparison test. 


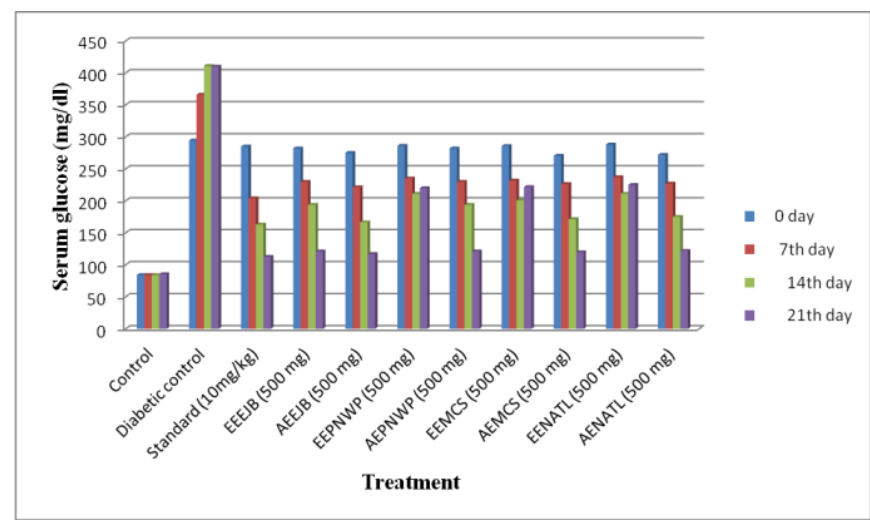

Graph 6: Effect of administration of feeding the ethanolic and aqueous extract of selected herb on serum glucose estimation in normal and diabetic rats

In the developing countries like India the prevalence of diabetes are very common and alarming. In traditional system of medicine herbal healers treat these diseases using the plants which have immense medicinal potentiality. Despite various available allopathic formulations, the relief from the disease is temporarily and has some side effects if used for the longer duration. The present work was undertaken to investigate anti-diabetic activity of selected herbs viz., EJB: Eugenia jambolana (Bark), PNWP: Phyllanthus niruri (Whole plant), MCS: Momordica charantia (Seeds) and NATL: Nyctanthes arbor-tristis (Leaves). From the results it was concluded that the aqueous extract showed better efficacy than the ethanolic extract in all the treated extract in lowering the blood glucose levels in alloxan induced diabetic rats.

\section{REFERENCES}

1. Ramachandran A, Snehalatha C, Viswanathan V. Burden of type 2 diabetes and its complications- the Indian scenario. Curr. Sci. 2002; 83:1471-1476.

2. Matteucci E, Giampietro O. Oxidative stress in families of type 1 diabetic patients. Diabetes Care. 2000; 23:1182-1186.

3. Baynes JW, Thorpe SR. The role of oxidative stress in diabetic complications. Curr. Opin. Endocrinol. 1997; 3:277-284.

4. Lipinski B. Pathophysiology of oxidative stress in diabetes mellitus. J. Diabet. Complications. 2001; 15:203-210.

5. Grover JK, Yadav S, Vats V. Medicinal plants of India with antidiabetic potential. J. Ethnopharmacol. 2002; 81:81-100.

6. Seth SD, Sharma B. Medicinal plants of India. Indian J. Med. Res. 2004; 120:9-11.

7. Ahmed R, Mostafa MN, Rahmatullah M. Oral glucose tolerance test (OGTT) with a combination of Colocasia esculenta stems and Eichhornia crassipes aerial parts. World J Pharm Pharm Sci. 2018; 7:207-214.

8. Ghosh D, Mandal I, Rumi JF, Trisha UK, Jannat H, Ahmed M et al. Effect of Allium sativum leaf extracts on glucose tolerance in glucose-induced hyperglycemic mice. Adv Nat Appl Sci. 2014; 8:66-69.

9. Islam MH, Mostafa MN, Rahmatullah M. Antihyperglycemic activity of methanolic extracts of corms of Colocasia esculenta var esculenta. Eur J Pharm Med Res. 2018; 5:129-132.

10. Joy KL, Kuttan RJ. Anti-diabetic activity of Picrorrhiza kurroa extract. J Ethnopharmacol. 1999; 67:143-148.

HOW TO CITE THIS ARTICLE: Nagar R, Patel R. Anti-diabetic Activity of Few India Indigenous Medicinal Plants. Int. J. Pharm. Sci. Drug Res. 2019; 11(6): 278-283. DOI: 10.25004/IJPSDR.2019.110601 